\title{
SYNTHESIS, ANTIMICROBIAL AND CYTOTOXIC ACTIVITIES OF 5-BENZYLIDENE-2-[(PYRIDINE- 4-YLMETHYLENE)HYDRAZONO]-THIAZOLIDIN-4-ONE AND 2-[(PYRIDINE-4-YLMETHYLENE) HYDRAZONO]-THIAZOLIDIN-4-ONE DERIVATIVES
}

\author{
Danniel Delmondes Feitoza, Antônio José Alves e José Gildo de Lima* \\ Departamento de Ciências Farmacêuticas, Universidade Federal de Pernambuco, Cidade Universitária, 50470-521 Recife - PE, Brasil \\ Janete Magali Araújo, Jaciana Santos Aguiar, Maria do Desterro Rodrigues, Teresinha Gonçalves Silva, Silene Carneiro \\ do Nascimento e Alexandre José da Silva Góes \\ Departamento de Antibióticos, Universidade Federal de Pernambuco, Cidade Universitária, 50670-901 Recife - PE, Brasil
}

Recebido em 27/4/11; aceito em 16/10/11; publicado na web em 20/1/12

\begin{abstract}
A new series of 5-benzylidene-2-[(pyridine-4-ylmethylene)hydrazono]-thiazolidin-4-ones 4a-I have been synthesized. These compounds were designed by a molecular hybridization approach. 2-[(Pyridine-4-ylmethylene)hydrazono]-thiazolidin-4-ones 3a-d were also obtained and used as intermediates to give the target compounds. The in vitro antimicrobial and cytotoxic activities were evaluated for both series. The intermediate $\mathbf{3 b}$ showed considerable antibiotic activity against B. subtilis and $C$. albicans. In the cytotoxic activity compounds $\mathbf{3 b}\left(\mathrm{IC}_{50}=4.25 \pm 0.36 \mu \mathrm{g} / \mathrm{mL}\right)$ and $\mathbf{4 l}\left(\mathrm{IC}_{50}=1.38 \pm 0.04 \mu \mathrm{g} / \mathrm{mL}\right)$ were effective for inhibition of human erythromyeloblastoid leukemia (K-562) and human lung carcinoma (NCI-H292) cell lines, respectively.
\end{abstract}

Keywords: 4-thiazolidinone; molecular hybridization; antimicrobial and cytotoxic activities.

\section{INTRODUCTION}

The emergence of antibiotic resistant bacteria can be regarded as a natural manifestation, governed by the principle of evolutionary genetic adaptation of microorganisms to changes in their environment. In fact, bacteria have shown success in its ability to develop resistance to multiple drugs. ${ }^{1}$ Recent studies have shown an increase of antibiotic resistance by Gram-positive bacterial strains, and a resistance has been detected to antibiotics ranging from the $\beta$-lactams, macrolides and quinolones to the glycopeptides and oxazolidinones., ${ }^{2,3}$ Due to this alarming fact, the search for drugs that provide a treatment more effective and safe has increased. This scenario leads us to develop new antibacterial agents active against microorganisms that have become resistant to existing drugs.

Furthermore, multidrug resistance (MDR) is one of the main obstacles in the cancer chemotherapy. MDR can be defined as the intrinsic or acquired resistance of cancer cells to multiple classes of structurally and mechanistically unrelated antitumor drugs. Circumvention of multidrug resistance is a new field of investigation in cancer chemotherapy, and safe and potent multidrug resistance inhibitors are needed for clinical use. ${ }^{4}$

4-Thiazolidinone ring system is a core structure in various biological active compounds with a wide range of biological and pharmacological properties such as anticancer and antimicrobial activities. ${ }^{5}$ Indeed, Vicini et al. ${ }^{6}$ synthesized and tested a series of 2-thiazolylimino-5-arylidene-4-thiazolidinone derivatives (a, Figure 1) and their precursors 2-thiazolylimino-4-thiazolidinone for antimicrobial activity against Gram positive and Gram negative bacteria, yeasts and mould. The authors highlighted that the substituted and unsubstituted 5-arylidene moiety plays an important role in enhancing the antimicrobial properties of this class of compounds. In turn, recent work in our research group showed that some 4-thiazolidinone derivatives (b, Figure 1) obtained from formilpyridine thiosemicarbazones possess considerable antimicrobial activity against Micrococcus luteus, Mycobacterium phlei, Mycobacterium smegmatis, Mycobacterium

*e-mail: jgildolima@gmail.com tuberculosis, Malassezia furfur and Candida albicans. ${ }^{7}$ On the other hand, Zhou et al. ${ }^{8}$ identified ten cytoselective compounds from 372 thiazolidinone analogues (c, Figure 1) by applying iterative library approaches. These compounds selectively killed both non-small cell lung cancer cell line $\mathrm{H} 460$ and its paclitaxel-resistant variant $\mathrm{H} 460 \operatorname{taxR}$ at an $\mathrm{IC}_{50}$ between 0.21 and $2.93 \mu \mathrm{M}$ while showing much less toxicity to normal human fibroblasts at concentrations up to $195 \mu \mathrm{M}$. Structure-activity relationship studies revealed that the nitrogen atom on the 4-thiazolidinone ring cannot be substituted; several substituents R" are tolerated at various positions; and the substituent $\mathrm{R}$ "' is restricted to the $-\mathrm{NMe}_{2}$ group at the 4-position.
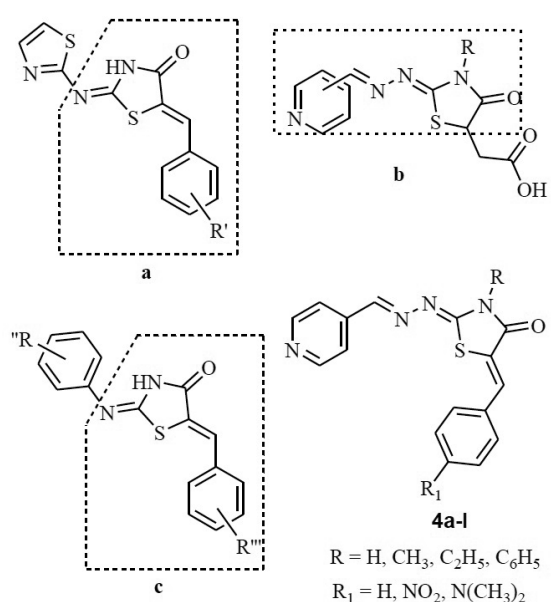

Figure 1. Design of the compounds $\mathbf{4 a - 1}$ using molecular hybridization approach

Based on the above results, in this paper we report the synthesis and antimicrobial activity of the 5-benzylidene-2-[(pyridine-4-ylmethylene)hydrazono]-thiazolidin-4-ones $\mathbf{4 a - 1}$ and their precursors 2-[(pyridine-4-ylmethylene)hydrazono]-thiazolidin-4-ones 3a-d (Scheme 1) against Gram positive and Gram negative bacteria and fungi. In addition, the cytotoxic properties of these compounds were 
tested against three strains of human cancer cells. Among the main strategies used in planning a series of structural analogues from a prototype, it was used the molecular hybridization for designing the compounds $\mathbf{4 a - 1}$. Thus, from this process we seek to merge the pyridinemethylenehydrazono-4-thiazolidinone moiety in $\mathbf{b}$ with the 5-benzylidene-4-thiazolidinone group present in a and $\mathbf{c}$ (Figure 1). So, we also hope to find new hybrid 4-thiazolidinone compounds that have either antimicrobial or anticancer activities.

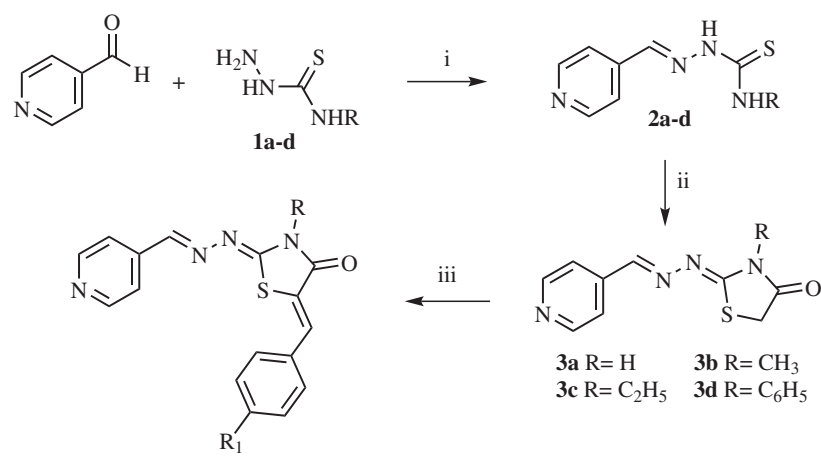

4a $\mathrm{R}=\mathrm{H}, \mathrm{R}_{1}=\mathrm{H}$ $4 b \mathrm{R}=\mathrm{H}, \mathrm{R}_{1}=\mathrm{NO}_{2}$ 4c R=H, R $=\mathrm{N}\left(\mathrm{CH}_{3}\right)_{2} \quad$ 4i $\mathrm{R}=\mathrm{C}_{2} \mathrm{H}_{5}, \mathrm{R}_{1}=\mathrm{N}\left(\mathrm{CH}_{3}\right)_{2}$ 4d $\mathrm{R}=\mathrm{CH}_{3}, \mathrm{R}_{1}=\mathrm{H} \quad 4 \mathrm{j} \mathrm{R}=\mathrm{C}_{6} \mathrm{H}_{5}, \mathrm{R}_{1}=\mathrm{H}$ $4 \mathbf{R}=\mathrm{CH}_{3}, \mathrm{R}_{1}=\mathrm{NO}_{2} \quad 4 \mathbf{k} \mathrm{R}=\mathrm{C}_{6} \mathrm{H}_{5}, \mathrm{R}_{1}=\mathrm{NO}_{2}$ 4f $\mathrm{R}=\mathrm{CH}_{3}, \mathrm{R}_{1}=\mathrm{N}\left(\mathrm{CH}_{3}\right)_{2}$ 4l $\mathrm{R}=\mathrm{C}_{6} \mathrm{H}_{5}, \mathrm{R}_{1}=\mathrm{N}\left(\mathrm{CH}_{3}\right)_{2}$

Scheme 1. Reagents and conditions: (i) $\mathrm{H}_{2} \mathrm{O}, \mathrm{EtOH}$, drops of acetic acid, reflux, $1-3$ h, (68-89\%); (ii) $\mathrm{ClCH}_{2} \mathrm{CO}_{2} \mathrm{H}$, anhydrous AcONa, EtOH, reflux, 16-20 h (67-82\%); (iii) ArCHO, EtOH, piperidine (cat.), reflux, 3-6 h(47-82\%)

\section{EXPERIMENTAL}

\section{Chemistry}

The melting points were determined on Büchi-535 apparatus and are uncorrected. IR spectra were measured on Bruker IFS-66 IR spectrophotometer. NMR spectra were recorded on Unity Plus $300 \mathrm{MHz}$ - Varian spectrometer by using tetramethylsilane as an internal standard. HRMS were recorded on Varian MAT $711 \mathrm{spec}-$ trometer $70 \mathrm{eV}$ electron impact, Kratos prole spectrometer $70 \mathrm{eV}$ electron impact. The chemical shifts are reported in $\delta$ units, and coupling constants $(\mathrm{J})$ are reported in Hertz $(\mathrm{Hz})$. TLC development was conducted on $0.25 \mathrm{~mm}$ silica gel plates (60254, Merck).

\section{General synthetic procedure process for compounds 2a-d}

All thiosemicarbazones 2a-d were synthesized by mixing an aqueous solution of thiosemicarbazides 1a-d (3.0 mmol in $10 \mathrm{~mL})$, ethanolic solution of 4-pyridinecarboxaldehyde $(3.0 \mathrm{mmol}$ in $10 \mathrm{~mL})$ and few drops of acetic acid under reflux for $1-3 \mathrm{~h}$ with continuous stirring. After cooling, the precipitated compound was filtered and recrystallized from ethanol. 2a: Reaction time: $1 \mathrm{~h}$. White solid; yield 66\%; mp 235-236 ${ }^{\circ} \mathrm{C}$, Lit. ${ }^{9} 253-254^{\circ} \mathrm{C}$. Rf: 0.45 (ethyl acetate:hexane, 7:3). IR ( $\mathrm{KBr}) \mathrm{cm}^{-1}: \mathrm{v}: 3421\left(\mathrm{NH}_{2}\right), 3152(\mathrm{~N}-\mathrm{H}), 1563(\mathrm{C}=\mathrm{N})$, $1061(\mathrm{C}=\mathrm{S})$. 2b: Reaction time: $1.5 \mathrm{~h}$. Beige solid, yield $82 \%$, mp 234-236 ${ }^{\circ} \mathrm{C}$, Lit. ${ }^{10} 238-239^{\circ} \mathrm{C}$. Rf: 0.50 (ethyl acetate:hexane, 7:3). IR $(\mathrm{KBr}) \mathrm{cm}^{-1} \mathrm{v}: 3151\left(\mathrm{NHCH}_{3}\right), 2945(\mathrm{~N}-\mathrm{H}), 1518(\mathrm{C}=\mathrm{N}), 1021(\mathrm{C}=\mathrm{S})$. (2c): Reaction time: $1.5 \mathrm{~h}$. Yellow solid, yield $73 \%, \mathrm{mp} 223-224{ }^{\circ} \mathrm{C}$, Lit. ${ }^{10} 228-229^{\circ} \mathrm{C}$. Rf: 0.50 (ethyl acetate:hexane, 8:2). IR $(\mathrm{KBr}) \mathrm{cm}^{-1}$ v: $3353\left(\mathrm{NHCH}_{2} \mathrm{CH}_{3}\right), 2980(\mathrm{~N}-\mathrm{H}), 1526(\mathrm{C}=\mathrm{N}), 1103(\mathrm{C}=\mathrm{S}) .2 d$ : Reaction time: $3 \mathrm{~h}$. Yellow solid, yield 81\%, mp 196-198 ${ }^{\circ} \mathrm{C}$, Lit. ${ }^{11}$ $200{ }^{\circ} \mathrm{C}$. Rf: 0.50 (ethyl acetate:hexane, 6:4). IR (KBr) $\mathrm{cm}^{-1}$ : v: 3306 $\left(\mathrm{NHCH}_{6} \mathrm{H}_{5}\right), 3106(\mathrm{~N}-\mathrm{H}), 1550(\mathrm{C}=\mathrm{N}), 1189(\mathrm{C}=\mathrm{S})$.

\section{General synthetic procedure process for compounds 3a-d}

A solution of $1.70 \mathrm{mmol}$ of thiosemicarbazones $\mathbf{2 a - d}, 1.90 \mathrm{mmol}$ of 2-chloroacetic acid, and $6.9 \mathrm{mmol}$ of sodium acetate anhydrous in $20 \mathrm{~mL}$ of ethanol was stirred until reflux till the completion of the reaction $(16-20 \mathrm{~h})$. After, the solution was cooled to $0{ }^{\circ} \mathrm{C}$, and the precipitate was collected with filter under vacuum and washed with hot methanol and water. 3a: Reaction time: $16 \mathrm{~h}$. White solid; yield 67\%; mp 262-263 ${ }^{\circ} \mathrm{C}$, Lit. ${ }^{12} 271^{\circ} \mathrm{C}$. Rf: 0.55 (ethyl acetate:hexane, 7:3). IR (KBr) cm${ }^{-1}$ : v: $1720(\mathrm{NC}=\mathrm{O}), 1633$ and $1550(\mathrm{C}=\mathrm{N}), 1290$ (NCS), 1230 (N-N=C), 1058 (CS); ${ }^{1} \mathrm{H}$ NMR (300 MHz, DMSO-d ${ }_{6}$ ) $\delta: 12.08$ (s, 1H, N-H), 8.64 (d, $J=6.0 \mathrm{~Hz}, 2 \mathrm{H}, \mathrm{Py}-\mathrm{H}), 8.40$ (s, $1 \mathrm{H}$, $\mathrm{CH}=\mathrm{N}), 7.65(\mathrm{~d}, J=6.0 \mathrm{~Hz}, 2 \mathrm{H}, \mathrm{Py}-\mathrm{H}), 3.90\left(\mathrm{~s}, 2 \mathrm{H}, \mathrm{S}-\mathrm{CH}_{2}\right) ;{ }^{13} \mathrm{C}$ NMR (75.4 MHz, DMSO-d $\left.)_{6}\right) \delta: 174.10(\mathrm{C}=\mathrm{O}), 167.80(\mathrm{~N}=\mathrm{C}), 154.20$ $(\mathrm{CH}=\mathrm{N}), 150.20$ (Py), 141.10 (Cq-Py), $121.20(\mathrm{Py}), 33.10\left(\mathrm{CH}_{2}-\mathrm{S}\right)$. HRMS (EI+): calcd. $\left(\mathrm{C}_{9} \mathrm{H}_{8} \mathrm{~N}_{4} \mathrm{OS}\right) 220.0419$; found: $220.0379 .3 \mathbf{b}$ : Reaction time: $16 \mathrm{~h}$. Yellow solid, $82 \%$; mp 146-148 ${ }^{\circ} \mathrm{C}$. Rf: 0.50 (ethyl acetate:hexane, 7:3). IR (KBr) $\mathrm{cm}^{-1}: \mathrm{v}: 1713(\mathrm{NC}=\mathrm{O}), 1625$ and $1568(\mathrm{C}=\mathrm{N}), 1302(\mathrm{NCS}), 1120(\mathrm{~N}-\mathrm{N}=\mathrm{C}), 1044(\mathrm{CS}) ;{ }^{1} \mathrm{H}$ NMR $\left(300 \mathrm{MHz}, \mathrm{DMSO}-\mathrm{d}_{6}\right) \delta: 8.67$ (d, $\left.J=4.5 \mathrm{~Hz}, 2 \mathrm{H}, \mathrm{Py}-\mathrm{H}\right), 8.52(\mathrm{~s}$, $1 \mathrm{H}, \mathrm{CH}=\mathrm{N}), 7.70(\mathrm{~d}, J=3.9 \mathrm{~Hz}, 2 \mathrm{H}, \mathrm{Py}-\mathrm{H}), 4.25\left(\mathrm{~s}, 3 \mathrm{H}, \mathrm{CH}_{3}\right), 4.0$ (s, 2H, S- $\left.\mathrm{CH}_{2}\right) ;{ }^{13} \mathrm{C}$ NMR (75.4 MHz, DMSO-d $)$ ) $8: 172.20(\mathrm{C}=\mathrm{O})$, $167.30(\mathrm{C}=\mathrm{N}), 155.40(\mathrm{CH}=\mathrm{N}), 150.00(\mathrm{Py}), 141.20(\mathrm{Cq}-\mathrm{Py}), 121.40$ (Py), $32.10\left(\mathrm{~S}_{-} \mathrm{CH}_{2}\right), 29.30\left(\mathrm{CH}_{3}\right)$; HRMS (EI+): calcd. $\left(\mathrm{C}_{10} \mathrm{H}_{10} \mathrm{~N}_{4} \mathrm{OS}\right)$ 234.0575; found: 234.0533 .3c: Reaction time: 16 h. Dark green solid; yield $75 \%$; mp $139-141^{\circ} \mathrm{C}$. Rf: 0.45 (ethyl acetate:hexane, $\left.1: 1\right)$. IR $(\mathrm{KBr}) \mathrm{cm}^{-1}$ : v. $1715(\mathrm{NC}=\mathrm{O}), 1615$ and $1515(\mathrm{C}=\mathrm{N}), 1315(\mathrm{NCS})$, $1235(\mathrm{~N}-\mathrm{N}=\mathrm{C}), 1088(\mathrm{CS}) ;{ }^{1} \mathrm{H}$ NMR (300 MHz, DMSO-d $\left.{ }_{6}\right) \delta: 8.67$ $(\mathrm{d}, J=5.7 \mathrm{~Hz}, 2 \mathrm{H}, \mathrm{Py}-\mathrm{H}), 8.52(\mathrm{~s}, 1 \mathrm{H}, \mathrm{CH}=\mathrm{N}), 7.69(\mathrm{~d}, J=6.0 \mathrm{~Hz}$, $2 \mathrm{H}, \mathrm{Py}-\mathrm{H}$ ), 4.00 (s, 2H, S-CH $), 3.75$ (q, $2 \mathrm{H}, J=7.2 \mathrm{~Hz}, \mathrm{CH}_{2} \mathrm{CH}_{3}$ ), $1.20\left(\mathrm{t}, J=7.2 \mathrm{~Hz}, 3 \mathrm{H}, \mathrm{CH}_{2} \mathrm{CH}_{3}\right) ;{ }^{13} \mathrm{C} \mathrm{NMR}\left(75.4 \mathrm{MHz}, \mathrm{DMSO}-\mathrm{d}_{6}\right)$ $\delta: 171.80(\mathrm{C}=\mathrm{O}), 166.40(\mathrm{C}=\mathrm{N}), 155.40(\mathrm{CH}=\mathrm{N}), 150.20(\mathrm{Py}), 140,90$ (Cq-Py), 121.20 (Py), $37.70\left(\mathrm{~N}-\mathrm{CH}_{2}\right), 32.10\left(\mathrm{~S}^{-} \mathrm{CH}_{2}\right), 12.10\left(\mathrm{CH}_{3}\right)$; HRMS (EI+): calcd. $\left(\mathrm{C}_{11} \mathrm{H}_{12} \mathrm{~N}_{4} \mathrm{OS}\right)$ 248.0732; found: 248.0598. 3d: Reaction time: $20 \mathrm{~h}$. Yellow solid; yield $70 \%$; mp 252-253 ${ }^{\circ} \mathrm{C}$. Rf: 0.55 (ethyl acetate:hexane, 6:4). IR $(\mathrm{KBr}) \mathrm{cm}^{-1}: \mathrm{v}: 1720(\mathrm{NC}=\mathrm{O})$, 1620 and $1535(\mathrm{C}=\mathrm{N}), 1380(\mathrm{NCS}), 1230(\mathrm{~N}-\mathrm{N}=\mathrm{C}), 1035$ (CS); ${ }^{1} \mathrm{H}$ NMR (300 MHz, DMSO-d ${ }_{6}$ ) $\delta: 8.70$ (d, $\left.J=4.7 \mathrm{~Hz}, 2 \mathrm{H}, \mathrm{Py}-\mathrm{H}\right)$, $8.40(\mathrm{~s}, 1 \mathrm{H}, \mathrm{CH}=\mathrm{N}), 7.65(\mathrm{~d}, J=4.7 \mathrm{~Hz}, 2 \mathrm{H}, \mathrm{Py}-\mathrm{H}), 7.41-7.60(\mathrm{~m}$, $5 \mathrm{H}, \mathrm{Ar}-\mathrm{H}), 4.18$ (s, 2H, S-CH $\mathrm{CH}_{2}$; HRMS (EI+): calcd. $\left(\mathrm{C}_{15} \mathrm{H}_{12} \mathrm{~N}_{4} \mathrm{OS}\right)$ 296.0732; found: 296.0600.

\section{General synthetic procedure process for compounds 4a-l}

A solution of $0.34 \mathrm{mmol}$ of 4-thiazolidinones 3a-d, $0.34 \mathrm{mmol}$ of aldehyde aromatic, and two drops of piperidine in $10 \mathrm{~mL}$ of ethanol was stirred until reflux till the completion of the reaction (3-6 h). After, the solution was cooled to $0{ }^{\circ} \mathrm{C}$, and the precipitate was collected with filter under vacuum and washed with hot ethanol and water. 4a: Reaction time: $4 \mathrm{~h}$. Yellow solid; yield 60\%; mp 282$283^{\circ} \mathrm{C}$. Rf: 0.45 (ethyl acetate:hexane, 7:3. IR $(\mathrm{KBr}) \mathrm{cm}^{-1}$ : v: 1715 $(\mathrm{NC}=\mathrm{O}), 1640$ and $1503(\mathrm{C}=\mathrm{N}), 1572(\mathrm{C}=\mathrm{C}), 1310(\mathrm{NCS}), 1235$ $(\mathrm{N}-\mathrm{N}=\mathrm{C}), 1050(\mathrm{CS}) ;{ }^{1} \mathrm{H}$ NMR (300 MHz DMSO-d $\left.{ }_{6}\right) \delta: 12.75$ (s, $1 \mathrm{H}, \mathrm{NH}), 8.70(\mathrm{~d}, J=5.4 \mathrm{~Hz}, 2 \mathrm{H}, \mathrm{Py}-\mathrm{H}), 8.55(\mathrm{~s}, 1 \mathrm{H}, \mathrm{CH}=\mathrm{N}), 7.80$ (d, $J=5.4 \mathrm{~Hz}, 2 \mathrm{H}, \mathrm{Py}-\mathrm{H}), 7.65$ (s, 1H, CH=C), 7.40-7.60 (m, 5H, Ar-H); HRMS(EI+): calcd. $\left(\mathrm{C}_{16} \mathrm{H}_{12} \mathrm{~N}_{4} \mathrm{OS}\right) 308.0732$; found: 308.0711 . 4b: Reaction time: $3 \mathrm{~h}$. Yellow solid; yield $82 \%$; $\mathrm{mp} 300^{\circ} \mathrm{C}$. Rf: 0.50 (ethyl acetate:hexane, 7:3). IR ( $\mathrm{KBr}) \mathrm{cm}^{-1}$ : v: $1708(\mathrm{NC}=\mathrm{O}), 1625$ and $1500(\mathrm{C}=\mathrm{N}), 1581(\mathrm{C}=\mathrm{C}), 1340(\mathrm{NCS}), 1300(\mathrm{~N}-\mathrm{N}=\mathrm{C}), 1007$ (CS); ${ }^{1} \mathrm{H}$ NMR (300 MHz DMSO-d $)$ ) $\delta .71$ (d, $J=5.7 \mathrm{~Hz}, 2 \mathrm{H}$, PyH), 8.55 (s, 1H, CH=N), 8.37 (d, J=9.0 Hz, 2H, Ar-H), 7.94 (d, 9.0 $\mathrm{Hz}, 2 \mathrm{H}, \mathrm{Ar}-\mathrm{H}), 7.70$ (s, 1H, CH=C), 7.76 (d, J = 5.7 Hz, 2H, Py-H); HRMS(EI+): calcd. $\left(\mathrm{C}_{16} \mathrm{H}_{11} \mathrm{~N}_{5} \mathrm{O}_{3} \mathrm{~S}\right)$ 353.0583; found: 353.0415. 4c: 
Reaction time: 5 h. Orange solid; yield 50\%; mp 252-253 ${ }^{\circ} \mathrm{C}$. Rf: 0.47 (ethyl acetate:hexane, 7:3). IR $(\mathrm{KBr}) \mathrm{cm}^{-1}: \mathrm{v}: 1714(\mathrm{NC}=\mathrm{O})$, 1647 and $1527(\mathrm{C}=\mathrm{N}), 1573(\mathrm{C}=\mathrm{C}), 1318(\mathrm{NCS}), 1250(\mathrm{~N}-\mathrm{N}=\mathrm{C})$, 1057 (CS); ${ }^{1} \mathrm{H}$ NMR (300 MHz DMSO-d 6 ) $\delta: 12.43$ (s, 1H, NH), $8.69(\mathrm{~d}, J=5.6 \mathrm{~Hz}, 2 \mathrm{H}, \mathrm{Py}-\mathrm{H}), 8.50(\mathrm{~s}, 1 \mathrm{H}, \mathrm{CH}=\mathrm{N}), 7.74(\mathrm{~d}, J=5.2$ $\mathrm{Hz}, 2 \mathrm{H}, \mathrm{Py}-\mathrm{H}), 7.61$ (s, 1H, CH=C), 7.51 (d, $J=8.0 \mathrm{~Hz}, 2 \mathrm{H}, \mathrm{Ar}-\mathrm{H})$, 6.81(d, $J=8.0 \mathrm{~Hz}, 2 \mathrm{H}, \mathrm{Ar}-\mathrm{H}), 3.02\left(\mathrm{~s}, 6 \mathrm{H}, \mathrm{N}\left(\mathrm{CH}_{3}\right)_{2}\right)$; HRMS(EI+): calcd. $\left(\mathrm{C}_{18} \mathrm{H}_{17} \mathrm{~N}_{5} \mathrm{OS}\right) 351.1154$; found: $351.1110 .4 \mathrm{~d}$ : Reaction time: 4 h. Dark yellow solid; yield 58\%; mp 162-164 ${ }^{\circ} \mathrm{C}$. Rf: 0.50 (ethyl acetate:hexane, 6:4). IR ( $\mathrm{KBr}) \mathrm{cm}^{-1}: \mathrm{v}: 1701(\mathrm{NC}=\mathrm{O}), 1620$ and $1536(\mathrm{C}=\mathrm{N}), 1565(\mathrm{C}=\mathrm{C}), 1374(\mathrm{NCS}), 1125(\mathrm{~N}-\mathrm{N}=\mathrm{C}), 1037(\mathrm{CS})$; ${ }^{1} \mathrm{H}$ NMR (300 MHz DMSO-d $\left.{ }_{6}\right) \delta: 8.72(\mathrm{~d}, J=5.4 \mathrm{~Hz}, 2 \mathrm{H}$, Py-H), $8.63(\mathrm{~s}, 1 \mathrm{H}, \mathrm{CH}=\mathrm{N}), 7.77(\mathrm{~d}, J=5.7 \mathrm{~Hz}, 2 \mathrm{H}, \mathrm{Py}-\mathrm{H}), 7.75(\mathrm{~s}, 1 \mathrm{H}$, $\mathrm{CH}=\mathrm{C}$ ), 7.50-7.70 (m, 5H, Ar-H), 2.55 (s, 3H, $\mathrm{CH}_{3}$ ); HRMS(EI+): calcd. $\left(\mathrm{C}_{17} \mathrm{H}_{14} \mathrm{~N}_{4} \mathrm{OS}\right) 322.0888$; found: $322.0727 .4 \mathrm{e}$ : Reaction time: 3 h. Dark yellow solid; yield 50\%; mp 266-268 ${ }^{\circ} \mathrm{C}$. Rf: 0.50 (ethyl acetate:hexane, 6:4). IR ( $\mathrm{KBr}) \mathrm{cm}^{-1}: \mathrm{v}: 1715(\mathrm{NC}=\mathrm{O}), 1624$ and $1535(\mathrm{C}=\mathrm{N}), 1567(\mathrm{C}=\mathrm{C}), 1340(\mathrm{NCS}), 1130(\mathrm{~N}-\mathrm{N}=\mathrm{C}), 1039(\mathrm{CS})$; ${ }^{1} \mathrm{H}$ NMR (300 MHz DMSO-d $\left.{ }_{6}\right) \delta: 8.74(\mathrm{~d}, J=5.6 \mathrm{~Hz}, 2 \mathrm{H}, \mathrm{Py}-\mathrm{H})$, 8.68 (s, $1 \mathrm{H}, \mathrm{CH}=\mathrm{N}), 8.37$ (d, $J=7.6 \mathrm{~Hz}, 2 \mathrm{H}, \mathrm{Ar}-\mathrm{H}), 7.97$ (d, $J=8.0$ $\mathrm{Hz}, 2 \mathrm{H}, \mathrm{Ar}-\mathrm{H}), 7.87$ (s, 1H, CH=C), 7.79 (d, J=5.6 Hz, 2H, Рy-H), 2.55 (s, 3H, $\left.\mathrm{CH}_{3}\right)$; HRMS(EI+): calcd. $\left(\mathrm{C}_{17} \mathrm{H}_{13} \mathrm{~N}_{5} \mathrm{O}_{3} \mathrm{~S}\right) 367.0739$; found: 367.0602 . 4f: Reaction time: 5 h. Purple solid; yield $47 \%$; mp 239-241 ${ }^{\circ} \mathrm{C}$. Rf: 0.45 (ethyl acetate:hexane, 7:3). IR (KBr) $\mathrm{cm}^{-1}$ : v: $1698(\mathrm{NC}=\mathrm{O}), 1598$ and $1530(\mathrm{C}=\mathrm{N}), 1566(\mathrm{C}=\mathrm{C}), 1326(\mathrm{NCS})$, $1200(\mathrm{~N}-\mathrm{N}=\mathrm{C}), 1115(\mathrm{CS}) ;{ }^{1} \mathrm{H}$ NMR $\left(300 \mathrm{MHz} \mathrm{CD}_{2} \mathrm{Cl}_{2}\right) \delta: 8.65(\mathrm{~d}$, $J=5.7 \mathrm{~Hz}, 2 \mathrm{H}, \mathrm{Py}-\mathrm{H}), 8.39(\mathrm{~s}, 1 \mathrm{H}, \mathrm{CH}=\mathrm{N}), 7.65(\mathrm{~d}, J=6.0 \mathrm{~Hz}, 2 \mathrm{H}$, Py-H), 7.61 (s, 1H, CH=C), 7.52 (d, J=8.7 Hz, 2H, Ar-H), 6.75 (d, $J=9.0 \mathrm{~Hz}, 2 \mathrm{H}, \mathrm{Ar}-\mathrm{H}), 3.77\left(\mathrm{~s}, 3 \mathrm{H}, \mathrm{CH}_{3}\right), 3.02\left(\mathrm{~s}, 6 \mathrm{H}, \mathrm{N}\left(\mathrm{CH}_{3}\right)_{2}\right)$; HRMS(EI+): calcd. $\left(\mathrm{C}_{19} \mathrm{H}_{19} \mathrm{~N}_{5} \mathrm{OS}\right) 365.1310$; found: $365.1274 . \mathbf{4 g}$ : Reaction time: $4 \mathrm{~h}$. Yellow solid; yield $78 \%$; mp 161-162 ${ }^{\circ} \mathrm{C}$. Rf: 0.50 (ethyl acetate:hexane, $1: 1)$. IR $(\mathrm{KBr}) \mathrm{cm}^{-1}: \mathrm{v}: 1700(\mathrm{NC}=\mathrm{O}), 1613$ and $1500(\mathrm{C}=\mathrm{N}), 1555(\mathrm{C}=\mathrm{C}), 1385$ (NCS), $1230(\mathrm{~N}-\mathrm{N}=\mathrm{C}), 1080(\mathrm{CS}) ;{ }^{1} \mathrm{H}$ NMR (300 MHz DMSO-d $\left.)_{6}\right) \delta: 8.75(\mathrm{~d}, J=5.5 \mathrm{~Hz}, 2 \mathrm{H}, \mathrm{Py}-\mathrm{H}), 8.68$ $(\mathrm{s}, 1 \mathrm{H}, \mathrm{CH}=\mathrm{N}), 7.82(\mathrm{~d}, J=5.5 \mathrm{~Hz}, 2 \mathrm{H}, \mathrm{Py}-\mathrm{H}), 7.87$ (s, $1 \mathrm{H}, \mathrm{CH}=\mathrm{C})$, 7.75-7.60 (m, $5 \mathrm{H}, \mathrm{Ar}-\mathrm{H}), 3.97$ (q, $\left.J=6.6 \mathrm{~Hz}, 2 \mathrm{H}, \mathrm{C}_{2} \mathrm{CH}_{3}\right), 1.30$ (t, $J$ $\left.=6.6 \mathrm{~Hz}, 3 \mathrm{H}, \mathrm{CH}_{2} \underline{\mathrm{CH}}_{3}\right)$; HRMS(EI+): calcd. $\left(\mathrm{C}_{18} \mathrm{H}_{16} \mathrm{~N}_{4} \mathrm{OS}\right) 336.1045$; found: $336.0870 . \mathbf{4 h}$ : Reaction time: 3 h. Dark red solid; yield $65 \%$; mp 244-246 ${ }^{\circ} \mathrm{C}$. Rf: 0.45 (ethyl acetate:hexane, $\left.1: 1\right)$. IR $(\mathrm{KBr}) \mathrm{cm}^{-1}$ : v: $1706(\mathrm{NC}=\mathrm{O}), 1613$ and $1500(\mathrm{C}=\mathrm{N}), 1555(\mathrm{C}=\mathrm{C}), 1385(\mathrm{NCS})$, $1230(\mathrm{~N}-\mathrm{N}=\mathrm{C}), 1080$ (CS); ${ }^{1} \mathrm{H}$ NMR (300 MHz DMSO-d 6 ) $\delta: 8.73$ (d, $J=5.7 \mathrm{~Hz}, 2 \mathrm{H}, \mathrm{Py}-\mathrm{H}), 8.68(\mathrm{~s}, 1 \mathrm{H}, \mathrm{CH}=\mathrm{N}), 8.37(\mathrm{~d}, J=8.7 \mathrm{~Hz}, 2 \mathrm{H}$, Ar-H), 7.95 (d, J=9.0 Hz, 2H, Ar-H), 7.86 (s, 1H, CH=C), 7.78 (d, $J=6.0 \mathrm{~Hz}, 2 \mathrm{H}, \mathrm{Py}-\mathrm{H}), 4.0\left(\mathrm{q}, J=6.9 \mathrm{~Hz}, 2 \mathrm{H}, \mathrm{CH}_{2} \mathrm{CH}_{3}\right), 1.30(\mathrm{t}, J=$ $\left.6.9 \mathrm{~Hz}, 3 \mathrm{H}, \mathrm{CH}_{2} \mathrm{CH}_{3}\right)$; HRMS(EI+): calcd. $\left(\mathrm{C}_{18} \mathrm{H}_{15} \mathrm{~N}_{5} \mathrm{O}_{3} \mathrm{~S}\right) 381.0896$; found: 381.0858. 4i: Reaction time: 5 h. Dark red solid; yield 53\%; mp 222-224 ${ }^{\circ} \mathrm{C}$. Rf: 0.45 (ethyl acetate:hexane, 4:6). IR (KBr) cm- ${ }^{1}$ : v: $1702(\mathrm{NC}=\mathrm{O}), 1630$ and $1520(\mathrm{C}=\mathrm{N}), 1555(\mathrm{C}=\mathrm{C}), 1350(\mathrm{NCS})$, $1200(\mathrm{~N}-\mathrm{N}=\mathrm{C}), 1035(\mathrm{CS}) ;{ }^{1} \mathrm{H} \mathrm{NMR}\left(300 \mathrm{MHz} \mathrm{CDCl}_{3}\right) \delta: 8.70(\mathrm{~d}$, $J=6.0 \mathrm{~Hz}, 2 \mathrm{H}, \mathrm{Py}-\mathrm{H}), 8.40(\mathrm{~s}, 1 \mathrm{H}, \mathrm{CH}=\mathrm{N}), 7.69(\mathrm{~d}, J=6.0 \mathrm{~Hz}, 2 \mathrm{H}$, Py-H), 7.67 (s, 1H, CH=C), 7.50 (d, J=9.0 Hz, 2H, Ar-H), 6.75 (d, $J=9.0 \mathrm{~Hz}, 2 \mathrm{H}, \mathrm{Ar}-\mathrm{H}), 4.00\left(\mathrm{q}, J=6.9 \mathrm{~Hz}, 2 \mathrm{H}, \mathrm{C}_{2} \mathrm{CH}_{3}\right), 3.10$ (s, $\left.6 \mathrm{H}, \mathrm{N}\left(\mathrm{CH}_{3}\right)_{2}\right), 1.35\left(\mathrm{t}, J=6.9 \mathrm{~Hz}, 3 \mathrm{H}, \mathrm{CH}_{2} \underline{\mathrm{C}}_{3}\right)$; $\mathrm{HRMS}(\mathrm{EI}+)$ : calcd. $\left(\mathrm{C}_{20} \mathrm{H}_{21} \mathrm{~N}_{5} \mathrm{OS}\right)$ 379.1467; found: 379.1284. 4j: Reaction time: 6 h. Yellow solid; yield 77\%; mp 262-263 ${ }^{\circ} \mathrm{C}$. Rf: 0.65 (ethyl acetate:hexane, 6:4). IR ( $\mathrm{KBr}) \mathrm{cm}^{-1}: \mathrm{v}: 1705(\mathrm{NC}=\mathrm{O}), 1616$ and $1500(\mathrm{C}=\mathrm{N}), 1570(\mathrm{C}=\mathrm{C}), 1370(\mathrm{NCS}), 1280(\mathrm{~N}-\mathrm{N}=\mathrm{C}), 1050(\mathrm{CS})$; ${ }^{1} \mathrm{H}$ NMR (300 MHz DMSO-d ${ }_{6}$ ) $\delta: 8.70$ (d, $\left.J=6.0 \mathrm{~Hz}, 2 \mathrm{H}, \mathrm{Py}-\mathrm{H}\right)$, 8.47 (s, 1H, CH=N), 7.81 (s, $1 \mathrm{H}, \mathrm{CH}=\mathrm{C}), 7.73$ (d, $J=6.0 \mathrm{~Hz}, 2 \mathrm{H}$, Py-H), 7.75-7.50 (m, 10H, Ar-H); HRMS(EI+): calcd. $\left(\mathrm{C}_{22} \mathrm{H}_{16} \mathrm{~N}_{4} \mathrm{OS}\right)$ 384.1045; found: $384.0890 .4 k$ : Reaction time: 4 h. Yellow solid; yield 69\%; mp 296-297 ${ }^{\circ} \mathrm{C}$. Rf: 0.50 (ethyl acetate:hexane, 6:4). IR (KBr) $\mathrm{cm}^{-1}$ : v: $1718(\mathrm{NC}=\mathrm{O}), 1620$ and $1500(\mathrm{C}=\mathrm{N}), 1575(\mathrm{C}=\mathrm{C}), 1385$
(NCS), 1290 (N-N=C), 1050 (CS); ${ }^{1} \mathrm{H}$ NMR (300 MHz DMSO-d $\left.{ }_{6}\right)$ $\delta: 8.69(\mathrm{~d}, J=5.6 \mathrm{~Hz}, 2 \mathrm{H}, \mathrm{Py}-\mathrm{H}), 8.49(\mathrm{~s}, 1 \mathrm{H}, \mathrm{CH}=\mathrm{N}), 8.38(\mathrm{~d}, J$ $=8.8 \mathrm{~Hz}, 2 \mathrm{H}, \mathrm{Ar}-\mathrm{H}), 8.0(\mathrm{~d}, J=8.4 \mathrm{~Hz}, 2 \mathrm{H}, \mathrm{Ar}-\mathrm{H}), 7.90(\mathrm{~s}, 1 \mathrm{H}$, $\mathrm{CH}=\mathrm{C}), 7.72(\mathrm{~d}, J=5.20 \mathrm{~Hz}, 2 \mathrm{H}, \mathrm{Py}-\mathrm{H}), 7.57-750(\mathrm{~m}, 5 \mathrm{H}, \mathrm{Ar}-\mathrm{H})$; HRMS(EI+): calcd. $\left(\mathrm{C}_{22} \mathrm{H}_{15} \mathrm{~N}_{5} \mathrm{O}_{3} \mathrm{~S}\right)$ 429.0896; found: 429.0732. 4l: Reaction time: 6 h. Yellow solid; yield $74 \%$; mp 220-221 ${ }^{\circ} \mathrm{C}$. Rf: 0.50 (ethyl acetate:hexane, 6:4). IR (KBr) cm ${ }^{-1}: \mathrm{v}: 1705(\mathrm{NC}=\mathrm{O}), 1615$ and $1535(\mathrm{C}=\mathrm{N}), 1580(\mathrm{C}=\mathrm{C}), 1370(\mathrm{NCS}), 1200(\mathrm{~N}-\mathrm{N}=\mathrm{C}), 1050$ (CS); ${ }^{1} \mathrm{H} \mathrm{NMR}\left(300 \mathrm{MHz} \mathrm{CDCl}_{3}\right) \delta: 8.65$ (d, $\left.J=5.4 \mathrm{~Hz}, 2 \mathrm{H}, \mathrm{Py}-\mathrm{H}\right)$, 8.28 (s, $1 \mathrm{H}, \mathrm{CH}=\mathrm{N}), 7.76(\mathrm{~s}, 1 \mathrm{H}, \mathrm{CH}=\mathrm{C}), 7.63(\mathrm{~d}, J=5.1 \mathrm{~Hz}, 2 \mathrm{H}$, Py-H), 7.50 (d, J=8.1 Hz, 2H, Ar-H), 7.56-7.41 (m, 5H, Ar-H), 6.80 (d, $J=8.4 \mathrm{~Hz}, 2 \mathrm{H}, \mathrm{Ar}-\mathrm{H}), 3.0$ (s, 6H, N($\left.\left(\mathrm{CH}_{3}\right)_{2}\right)$; HRMS(EI+): calcd. $\left(\mathrm{C}_{24} \mathrm{H}_{21} \mathrm{~N}_{5} \mathrm{OS}\right)$ 427.1467; found: 427.1294 .

\section{Biology}

Assay in vitro for antimicrobial activity

Bacteria and fungal species used in the antimicrobial evaluation were obtained from Departamento de Antibióticos and Instituto de Micologia cultures collections, Universidade Federal de Pernambuco, Brazil. Namely, Staphylococcus aureus (ATTC 6538), Bacillus subtilis (UFPEDA 16), Micrococcus luteus (ATTC 2225), Escherichia coli (ATTC 25922), Mycobacterium smegmatis (UFPEDA 71), Candida sp. (UFPEDA 1315), Candida albicans (UFPEDA 1007) and Aspergilus niger (UFPEDA 2003) species. The antibacterial and antifungal activities are reported preliminary utilizing disc diffusion method. ${ }^{13}$ In this method, disks containing known amounts of an antimicrobial agent were placed on the surface of an agar plate that has been inoculated with a standardized suspension of microorganisms tested. Paper discs with only DMSO were used as negative controls. The MZI (Mean Zone Inhibition) for chloramphenicol and rifampicin (antibacterial), and nistatin (antifungal) was referred to as a reference value $(\mathrm{mm})$. All experiments were carried out three times and repeated if the results differed. For MIC assays, ${ }^{14}$ a stock solution $(1 \mathrm{mg} / \mathrm{mL})$ of test compounds was prepared in DMSO solvent. Further, the serial dilution of test compounds was carried out and the concentrations used ranged from 10 for $1000 \mu \mathrm{g} / \mathrm{mL}$. Test compounds at various concentrations were added to culture medium in a test tube and different strains were inoculated at $10^{8}$ bacteria/ $\mathrm{mL}$ concentration. Tryptic Soy Agar was utilized for culture medium. The tubes were incubated at $37^{\circ} \mathrm{C}$ for $24 \mathrm{~h}$ and then examined for the presence or absence of growth organisms tested. The MIC values were obtained from the lowest concentration of the test compounds where the tubes remained clear, indicating that the bacterial or fungal growth was completely inhibited at this concentration. The MIC values were expressed in $\mu \mathrm{g} / \mathrm{mL}$.

\section{In vitro assay for cytotoxic activity}

The human lung carcinoma cell line (NCI-H292), the human larynx carcinoma cell line (HEp-2) and the human erythromyeloblastoid leukemia (K-562) were purchased from the Adolfo Lutz Institute, São Paulo, Brazil. A DMEM (Dulbecco's Modified Eagle's Medium), enriched with $10 \%$ of fetal bovine serum, $1 \%$ of L-glutamine and $1 \%$ of antibiotics (penicillin and streptomycin), was used for cell cultivation and to perform the tests. The cytotoxic activity was investigated using the MTT assay (3-(4,5-dimethylthiazole-2-yl)-2,5diphenyltetrazolium bromide). ${ }^{15}$ Cell suspensions were diluted to $10^{5}$ cells $/ \mathrm{mL}$, suitably prepared and distributed in plates of culture with 96 wells $\left(225 \mu \mathrm{L}\right.$ in each well), then incubated at $37^{\circ} \mathrm{C}$ in a humid atmosphere with $5 \%$ of $\mathrm{CO}_{2}$. After $24 \mathrm{~h}, 25 \mu \mathrm{L}$ of either the synthesized compounds or the reference drugs (methotrexate and etoposide) was added to each well. The plates were incubated again at $37{ }^{\circ} \mathrm{C}$ for $72 \mathrm{~h}$. Then, $25 \mu \mathrm{L}$ of MTT solution $(5 \mathrm{mg} / \mathrm{mL})$ was 
added to each well, and the mixture was incubated at $37^{\circ} \mathrm{C}$ for $2 \mathrm{~h}$. At the end of this period, the culture medium with the MTT excess was aspirated and after that, $100 \mu \mathrm{L}$ of DMSO was added to each well to dissolve the formazan crystals. The optical density (OD) of the wells was measured at $540 \mathrm{~nm}$ and compared to the control (cells with medium only). The data represent the mean of two experiments in triplicate and were expressed as means $\pm \mathrm{SD} . \mathrm{IC}_{50}, \mathrm{EC}_{50}$ and their 95\% confidence intervals were determined from nonlinear regression using the program SigmaPlot version 11 . The $\mathrm{IC}_{50}$ value was defined as the concentration at which $50 \%$ survival of cells was observed; $\mathrm{EC}_{50}$ value was defined as the effective concentration causing 50\% hemolysis. With only a few exceptions ( $3 \mathbf{b}$ for K-562 cell line and 41 for NCI-H292 cell line), all of the compounds exhibited poor inhibitory effect on the growth of the NCI-H292, HEp-2 and K-562 cell lines with $\mathrm{IC}_{50}>10 \mu \mathrm{g} / \mathrm{mL}$. Reference drugs, methotrexate and etoposide, showed the following $\mathrm{IC}_{50}: 1.31 \pm 0.03 \mu \mathrm{g} / \mathrm{mL}$ and 2.75 $\pm 0.1 \mu \mathrm{g} / \mathrm{mL}$ for NCI-H292 cell line; $>20 \mu \mathrm{g} / \mathrm{mL}$ and $6.10 \pm 0.19$ $\mu \mathrm{g} / \mathrm{mL}$ for HEp-2 cell line; $>20 \mu \mathrm{g} / \mathrm{mL}$ and $4.48 \pm 0.23 \mu \mathrm{g} / \mathrm{mL}$ for K-562 cell line.

\section{RESULTS AND DISCUSSION}

For the synthesis of the target compounds, the reaction sequences outlined in Scheme 1 were followed. The thiosemicarbazones 2a-d were synthesized by literature procedures by heating the equimolar amounts of 4-pyridinecarbaldehyde and suitable thiosemicarbazides 1a-d in water/ethanol media containing few drops of acetic acid as catalyst. ${ }^{16}$ The resulting mixtures were allowed to slowly evaporate and the resulting crystals were isolated by filtration. This protocol afforded the corresponding thiosemicarbazones 2a-d in 67-89\% yields. The structure of the obtained compounds was checked by IR spectra which were in agreement with literature data. ${ }^{9-11}$

The synthesis of 2-[(pyridine-4-ylmethylene)hydrazono]-thiazolidin-4-ones 3a-d was performed by condensation of 4-formylpyridine thiosemicarbazones $\mathbf{2 a - d}$ with 2-chloroacetic acid in the presence of sodium acetate anhydrous in ethanol, ${ }^{17}$ and isolation in good yield ranging from 67 to $82 \%$. Synthesized molecules 3a-d were structurally characterized by spectroscopic analysis. IR spectra revealed the presence of lactam $\mathrm{C}=\mathrm{O}$ stretching bands and NCS bending vibration of the ring at $1713-1720 \mathrm{~cm}^{-1}$ and $1302-1380 \mathrm{~cm}^{-1}$, respectively. This is considered to be a strong confirmation of the thiazolidinone nucleus formation. ${ }^{18}$ Further evidence for the formation of 4-thiazolidinones 3a-d was obtained from the ${ }^{1} \mathrm{H}$ NMR and ${ }^{13} \mathrm{C}$ NMR spectra. The ${ }^{1} \mathrm{H}$ NMR spectra revealed the presence of a single signal at 3.90-4.18 ppm corresponding to methylene moiety of thiazolidinone nucleus. ${ }^{19}$ On the other hand, peaks resonated in the range 32.10-33.00, 163.10167.80 and $171.80-174.10 \mathrm{ppm}$ in the ${ }^{13} \mathrm{C}$ NMR spectra were assigned for $\mathrm{S}-\mathrm{CH}_{2}, \mathrm{C}=\mathrm{N}$ and $\mathrm{C}=\mathrm{O}$ moieties. ${ }^{20,21}$

The final compounds 5-arylidene-4-thiazolidinones 4a-I were obtained by Knoevenagel condensation of the previous products with commercially available aromatic aldehydes (benzaldheyde, 4-nitrobenzaldehyde and 4-dimethylaminobenzaldehyde) in yields varying from 47 to $82 \%$. The reaction was carried out in ethanol at reflux with piperidine as catalyst. ${ }^{22}$ The chemical structures were established using IR, ${ }^{1} \mathrm{H}$ NMR and HRMS spectroscopy. The IR spectra of 4a-l showed absorption band corresponding to the stretching mode $v(\mathrm{C}=\mathrm{O})$ of the lactam group at about $1698-1718 \mathrm{~cm}^{-1}$. The exocyclic $\mathrm{C}=\mathrm{C}$ bond was also assigned by means of the IR spectra, which showed a stretching band in the region of $1555-1581 \mathrm{~cm}^{-1}$. In the ${ }^{1} \mathrm{H}$ NMR spectra, the introduction of 5-arylidene moiety was confirmed through the absence of the signal of $5-\mathrm{CH}_{2}$ protons of $\mathbf{3 a - d}$ and the presence of resonances assigned to the methine hydrogen appearing as singlet (7.61-7.90 ppm). Unfortunately, due to poor solubility of the compounds 4a-l it was not possible to record the ${ }^{13} \mathrm{C}$ NMR spectra. HRMS data fully agree with the proposed structure.

The compounds 3a-d and $\mathbf{4 a - 1}$ were preliminary tested to antimicrobial activity by the disc diffusion method against various bacteria and fungal species. The mean zone inhibition (MZI) for chloranphenicol and rifampicin (antibacterial), and nistatin (antifungal) was referred to as a reference value (in $\mathrm{mm}$ ). In general, the results indicated weak antimicrobial activities for all compounds (Table 1). Except for compound $\mathbf{3 b}$, none of the tested compounds displayed good inhibition of the growth (MZI above or equal to $18 \mathrm{~mm}$ ) of Gram positive and Gram negative bacteria, and fungi in relation to the reference drugs. Compound 3b showed significant MZI for Bacillus subtilis $(26 \mathrm{~mm})$ and for Candida albicans $(25 \mathrm{~mm})$, but this derivative demonstrated higher values of MIC ( $>500$ and $>250 \mu \mathrm{g} / \mathrm{mL}$, respectively) when compared with chloranphenicol $(\mathrm{MIC}=30 \mu \mathrm{g} / \mathrm{mL}$ ) and rifampicin $(\mathrm{MIC}=120 \mu \mathrm{g} / \mathrm{mL})$ and nistatin $(\mathrm{MIC}=50 \mu \mathrm{g} / \mathrm{mL})$.

Table 1. Inhibitory zone diameters $(\mathrm{mm})$ of $\mathbf{3 a - d}$ and $\mathbf{4 a - l}$ against tested bacterial and yeast strains by disc diffusion method

\begin{tabular}{|c|c|c|c|c|c|c|c|c|}
\hline \multirow{2}{*}{ compound } & \multicolumn{8}{|c|}{ Mean Zone Inhibition (MZI) in $\mathrm{mm}^{\mathrm{a}}$} \\
\hline & $S a$ & Bs & $M l$ & $E c$ & Ms & $\mathrm{Ca}$ & Cs & $A n$ \\
\hline 3a & - & 16 & - & - & 15 & 16 & - & 17 \\
\hline $3 \mathbf{b}$ & - & 26 & - & - & 10 & 25 & 14 & 16 \\
\hline $3 c$ & 15 & 17 & 17 & - & 15 & 16 & 9 & 16 \\
\hline 3d & - & - & 15 & - & - & 13 & 10 & - \\
\hline $4 \mathbf{a}$ & - & - & - & - & 16 & 16 & - & 15 \\
\hline $4 b$ & - & - & - & - & - & - & - & - \\
\hline $4 c$ & - & - & - & - & - & - & - & - \\
\hline $4 d$ & - & - & 16 & - & 15 & 15 & 12 & 16 \\
\hline $4 e$ & - & - & - & - & 8 & 17 & 7 & 16 \\
\hline $4 f$ & - & - & 15 & - & 7 & 17 & 10 & - \\
\hline $4 \mathrm{~g}$ & - & 14 & 15 & - & 16 & 16 & - & 15 \\
\hline $4 h$ & - & 8 & 16 & 13 & 17 & 17 & 10 & 9 \\
\hline $4 i$ & - & - & 15 & 13 & 13 & 17 & 11 & - \\
\hline $4 \mathbf{j}$ & 11 & - & 15 & - & 12 & 13 & 11 & - \\
\hline $4 k$ & - & - & 14 & - & - & 13 & 10 & - \\
\hline 41 & - & - & 14 & - & - & - & 10 & - \\
\hline Rifampicin & 31 & 20 & 49 & 14 & 30 & NT & NT & NT \\
\hline Chloranphenicol & 25 & 27 & 50 & 27 & NT & NT & NT & NT \\
\hline Nistatin & NT & NT & NT & NT & NT & 26 & 18 & 20 \\
\hline
\end{tabular}

${ }^{a}$ Values are mean $(\mathrm{n}=3)$. Rifampicin $(100 \mu \mathrm{g} /$ disc $)$, chloramphenicol $(100 \mu \mathrm{g} /$ disc) and nistatin $(100 \mu \mathrm{g} / \mathrm{disc})$ were used as positive reference; compounds 3a-d and 4a-l ( $100 \mu \mathrm{g} / \mathrm{disc})$. - indicates no sensitivity or MZI lower that $7 \mathrm{~mm}$. NT, not tested. Sa: Staphylococcus aureus, Bs: Bacillus subitis, Ml: Micrococcus luteus, Ec: Escherichia coli, Ms: Mycobacterium smegmatis, Ca: Candida albicans, Cs: Candida sp, An: Aspergilus niger.

Next, the antiproliferative activity of the compounds $\mathbf{3 a - d}$ and 4a-l was tested against the NCHI-H292 (mucoepidermoid carcinoma of lung), HEp-2 (mucoepidermoid carcinoma of larynx) and K562 (human erythromyeloblastoid leukemia) cell lines. ${ }^{15}$ The cytotoxic activities of new compounds were compared with the know antitumor agents, methotrexate (MTX) and etoposide. In general, the results indicated non-significant antiproliferative activity even at $10 \mu \mathrm{g} / \mathrm{mL}$. However, the inhibitory effect of compound $\mathbf{3 b}$ on the growth of the $\mathrm{K}-562$ cell line was similar to that of etoposide and greater than MTX with $\mathrm{IC}_{50}=4.25 \pm 0.36 \mu \mathrm{g} / \mathrm{mL}, \mathrm{IC}_{50}=4.48 \pm 0.23 \mu \mathrm{g} / \mathrm{mL}$ and $\mathrm{IC}_{50}>$ 
$20 \mu \mathrm{g} / \mathrm{mL}$, respectively. On the other hand, compound $4 \mathbf{l}$ showed excellent anticancer activity against NCI-H292 cell line with $\mathrm{IC}_{50}=$ $1.38 \pm 0.04 \mu \mathrm{g} / \mathrm{mL}$. This compound showed equipotent to $\mathrm{MTX}\left(\mathrm{IC}_{50}=\right.$ $1.31 \pm 0.03 \mu \mathrm{g} / \mathrm{mL})$ and more active than etoposide $\left(\mathrm{IC}_{50}=2.75 \pm 0.1\right.$ $\mu \mathrm{g} / \mathrm{mL})$. Moreover, the absence of hemolysis in mice erythrocytes $\left(\mathrm{EC}_{50}>500 \mu \mathrm{g} / \mathrm{mL}\right)$ suggests that the cytotoxicity is not related with damage on membrane.

From the viewpoint of preliminary structure-activity relationships, the main features could be summarized as follows: (1) unlike the results obtained by Vicini et $_{\text {al. }}{ }^{6}$ in 2-thiazolylimino-5-arylidene4-thiazolidinone derivatives (a, Figure 1), the presence of 5-arylidene moiety in the series 4a-l did not prove to be important for the antimicrobial activity; (2) according to the results reported by Zhou et $a l .{ }^{8}$ in analogue series (c, Figure 1), compounds with nitrogen substitutions on the 4-thiazolidinone did not show any cytoselective toxicity. Therefore, we found that this structural requirement maybe is not crucial in our series, because the compound $4 \mathrm{l}\left(\mathrm{R}=\mathrm{C}_{6} \mathrm{H}_{5}\right.$, $\mathrm{R}_{1}=\mathrm{NMe}_{2}$ ) was the only active against NCI-H292 cell line and the $\mathrm{N}$-unsubstitued compound $\mathbf{4 c}$ bearing $\mathrm{NMe}_{2}$ group at the 4-position in the 5-arylidene moiety presented no significant activity; (3) a comparison of the structure of the compounds $4 \mathbf{a}-\mathbf{l}$ with those of 2-arylimino-5-arylidene-4-thiazolidinone analogs (a and c, Figure 1) led us to suppose that the hydrazone moiety might be related to lack of both antimicrobial and anticancer activities for most compounds.

\section{CONCLUSIONS}

In conclusion, we have investigated the antimicrobial and cytotoxic properties of the 4-thiazolidinone scaffold, utilizing a series of 5-benzylidene-2-[(pyridine-4-ylmethylene)hydrazono]-thiazolidin-4-ones 4a-l and their precursors 2-[(pyridine-4-ylmethylene) hydrazono]-thiazolidin-4-ones $\mathbf{3 a - d}$. The series $\mathbf{4}$ was designed by molecular hybridization using the 2-arylimino-5-arylidene4-thiazolidinone and pyridinemethylenehydrazono-4-thiazolidinone derivatives as prototypes. The compounds were synthesized with good yields and characterized based on their physical, analytical and spectral data. The compounds were evaluated in vitro against bacteria and fungal species, showing weak antimicrobial activities. Also in vitro study, the inhibitory effect of compound $\mathbf{3 b}\left(\mathrm{IC}_{50}=4.25 \pm 0.36 \mu \mathrm{g} /\right.$ $\mathrm{mL}$ ) on the growth of the $\mathrm{K}-562$ cell line was equipotent to etoposide $\left(\mathrm{IC}_{50}=4.48 \pm 0.23 \mu \mathrm{g} / \mathrm{mL}\right)$. It is noteworthy that only compound $4 \mathrm{l}$ exhibited excellent cytotoxic activity against mucoepidermoid carcinoma of lung cell NCI-H292, with $\mathrm{IC}_{50}=1.38 \pm 0.04 \mu \mathrm{g} / \mathrm{mL}$. On the basis of the biological results so far acquired, we could identify some structural features in our series: the 5-arylidene moiety did not show to be essential for the antimicrobial activity; an aromatic ring at the nitrogen atom on the 4-thiazolidinone ring and the $-\mathrm{NMe}_{2}$ group at the 4-position in the 5-arylidene moiety can play an important role in the citotoxic properties of this class of compounds; and, at first sight, the hydrazone fragment seems be related to the lack of both cytotoxic and antimicrobial activities.

\section{SUPPLEMENTARY MATERIAL}

Available on the http://quimicanova.sbq.org.br, in pdf file, with free access.

\section{ACKNOWLEDGEMENTS}

The authors thank the Conselho Nacional de Desenvolvimento Científico e Tecnológico $(\mathrm{CNPq})$, the Fundação de Amparo à
Ciência e Tecnologia do Estado de Pernambuco (FACEPE) and the Pró-Reitoria de Pesquisa da UFPE (Propesq) for financial support, Analytical Center of DQF-UFPE for the NMR, IR, and MS spectra.

\section{REFERENCES}

1. Guimarães, D. D.; Momesso, L. S.; Pupo, M. T.; Quim. Nova 2010, 33, 667.

2. Gould, I. M.; Int. J. Antimicrob. Agents 2009, 34, S3, S2.

3. Byarugaba, D. K.; Int. J. Antimicrob. Agents 2004, 24, 105.

4. Avendano, C.; Menendez, J. C.; Curr. Med. Chem. 2002, 9, 159.

5. Verma, A.; Saraf, S. K.; Eur. J. Med. Chem. 2008, 43, 897; Liesen, A. P.; Aquino, T. M.; Carvalho, C. S.; Lima, V. T.; Araújo, J. M.; Lima, J. G.; Faria, A. R.; Melo, E. J. T.; Alves, A. J.; Alves, E. W.; Alves, A. Q.; Góes, A. J. S.; Eur. J. Med. Chem 2010, 45, 3685; Pan, B.; Huang, R.; Han, S.; Qu, D.; Zhu, M.; Wei, P.; Ying, H.; Bioorg. Med. Chem. Lett. 2010, 20, 2461; Vicini, P.; Geronikaki, A.; Incerti, M.; Zani, F.; Dearden, J.; Hewitt, M.; Bioorg. Med. Chem. 2008, 16, 3714; Kline, T.; Barry, K. C.; Jackson, S. R.; Felise, H. B.; Nguyen, H. V.; Miller, S. I.; Bioorg. Med. Chem. Lett. 2009, 19, 1340.

6. Vicini, P.; Geronikaki, A.; Kitka, A.; Incerti, M.; Zani, F.; Bioorg. Med. Chem. 2006, 14, 3859.

7. Verçoza, G. L.; Feitoza, D. D.; Alves, A. J.; Aquino, T. M.; Lima, J. G.; Araújo, J. M.; Cunha, I. G. B.; Góes, A. J. S.; Quim. Nova 2009, 32, 1405 .

8. Zhou, H.; Wu, S.; Zhai, S.; Liu, A.; Sun, Y.; Li, R.; Zhang, Y.; Ekins, S.; Swaan, P. W.; Fang, B.; Zhang, B.; Yan, B.; J. Med. Chem. 2008, 51, 1242 .

9. Mendes, I. C.; Teixeira, L. R.; Lima, R.; Beraldo, H.; Speziali, N. L.; West, D. X.; J. Mol. Struct. 2001, 559, 355.

10. Beraldo, H.; Teixeira, L. R.; Moura, A. A.; West, D. X.; J. Mol. Struct. 2001, 559, 99.

11. Grammaticakis, P.; Bull. Soc. Chim. Fr. 1956, 109.

12. Buu-Hoi, N. P.; Xuong, N. D.; Binon, F.; J. Chem. Soc. 1954, 713.

13. Bauer, A. W.; Kirby, W. M. M.; Scherris, J. C.; Truck, M.; Am. J. Clin. Path. 1966, 45, 493.

14. National Committee For Clinical Laboratory Standards; Methods for Dilution Antimicrobial Susceptibility Tests for Bacteria That Grow Aerobically, $3^{\text {rd }}$ ed., Approved Standard, NCCLS publication M7-A3: Villanova, 1993; National Committee for Clinical Laboratory Standards; Reference Method for Broth Dilution Antifungal Susceptibility Testing of Yeasts, Proposed Standard, NCCLS Document M27-P: Villanova, 1992.

15. Alley, M. C.; Scudiero, D. A.; Monsk, A.; Hursey, M. I.; Czerwinski, M. J.; Fine, D. L.; Abbott, B. J.; Mayo, J. G.; Shoemaker, R. H.; Boyd, M. R.; Cancer Res. 1988, 48, 589; Mosmann, T.; J. Immunol. Methods 1983, 16, 55 .

16. Tenório, R. P.; Carvalho, C. S.; Pessanha, C. S.; Lima, J. G.; Faria, A. R.; Alves, A. J.; Melo, E. J. T.; Góes, A. J. S.; Bioorg. Med. Chem. Lett. 2005, 15, 2575.

17. Bonde, C. G.; Gaikwad, N. J.; Bioorg. Med. Chem. 2004, 12, 2151.

18. Çapan, G.; Ulusoy, N.; Ergenç, N.; Kiraz, M.; Monatsh. Chem. 1999, 130, 1399.

19. Ergenç, N.; Capan, G.; Günay, N. S.; Özkirimli, S.; Güngör, M.; Özbey, S.; Kendi, E.; Arch. Pharm. 1999, 332, 343.

20. Küçükgüzel, S. G.; Oruç, E. E.; Rollas, S.; Sahin, F.; Özbek, A.; Eur. J. Med. Chem. 2002, 37, 197.

21. Küçükgüzel, G.; Kocatepe, A.; Clercq, E.; Sahin, F.; Güllüce, M.; Eur. J. Med. Chem. 2006, 41, 353.

22. Ottaná, R.; Maccari, R.; Ciurleo, R.; Paoli, P.; Jacomelli, M.; Manao, G.; Camici, G.; Laggner, C.; Langer, T.; Bioorg. Med. Chem. 2009, 17, 1928. 